\title{
Attainable Throughput Determination for Cognitive Radio
}

\author{
M. Ajay Kumar ${ }^{1}$, Dr.N.Rajesha ${ }^{2}$, Dr. Anupama Deshpande ${ }^{3}$ \\ ${ }^{1}$ Research Scholar, Dept of ECE, Shri JJT University, Jhunjhunu-01 \\ ${ }^{2}$ Associate Professor, Dept of ECE, Malla Reddy Institute of Engg and Tech, Hyderabad-14 \\ ${ }^{3}$ Professor, Dept of ECE, Shri JJT University, Jhunjhunu-01 \\ Email: ajayajju126@gmail.com ${ }^{1}$,rajeshmurthy44@gmail.com ${ }^{2}$,Mangala.d.2000@gmail.com ${ }^{3}$
}

\begin{abstract}
In mechanism of cognitive-radio networks, typical cognitive secondary users were authorized to use the primary user bands, in a specified case when the band is in idle condition which means unused. For this effective mechanism, the cognitive unlicensed users (secondary users) are essential to sense frequency environment, as well as the licensed user (primary user) is active, and also secondary users has to void the channel in specified time. The channel sensing parameters are: false alarm detection and detection probability. When the detection probability is higher, the more the cognitive primary users are protected. Nevertheless, in perspective of secondary users', typical probability of false alarm is lower; the better an chance for reusing the channel, thence the attainable throughput is higher for the secondary-users. In this specific paper, we analyze the essential tradeoff among achievable throughput and sensing capability of secondaryusers. In addition to, we represent the framework for the sensing of slot duration to enlarge the throughput which is attainable for secondary-users below the constraints which the cognitive primary-users are effectively protected. By utilizing the energy spotting scheme, the optimum sensing time that gives the better tradeoff is inclined. The co-operative sensing is analyzed with proper methodology of suggested sensing throughput-tradeoff. The simulations are conferred to estimate the suggested trade off methodology.
\end{abstract}

Index Terms-Cognitive radio networks, Throughput, Primary users, Wireless communications.

\section{INTRODUCTION}

The spectrum scarcity is because of increase in demand for bans of spectrum; this issue is solved by the increase in usage of spectrum that is accomplished by admitting cognitive unlicensedusers to use the frequency band, when licensedusers don't utilize it. The typical CRNs are proposed for using the band of spectrum more effectively. The most critical mechanism in CR is typical spectrum sensing, this mechanism has distinct sensing approaches. From these the effective approach is energy spotting and the alternative approaches were characterized in[2], moreover the energy spotting model is contemplated in this specific research because of its compelling factors and no priori data about cognitive PUs signals. Accordingly, in co-operative sensing and local sensing it's been studied significantly.

In typical cooperative sensing, the data regarding the detection of cognitive PUs is sensed. In the CR network which is centralized, a prevailing receiver plays the critical-role in the data collection and sensing of white spaces.

In this particular paper, the false alarm anticipation effect is determined and detection probability on through-put regarding the secondary-networks. The conventional star-topology regarding is considered for secondary networks; moreover the time for propagation takes for outcomes of the local-sensing in distinction to individual user has delay; It represented by determines the throughput regarding cognitive secondary user declines by the delay of cooperation that increases as sum of the cognitive users increases.

This specific paper has formulated as following. Portion 2 represents the typical system-model regarding spectrum sensing mechanism. The typical network scheme and cooperation method is rooted in this distinct portion. In portion 3 , we focused the systematic theory about the effective spectrum sensing as well as co-operative sensing dependent on energy spotting approach and analyzes the cooperation overhead for cooperative sensing. The throughput of secondary network; portion 4 presents the simulation results dependent on analytic model and portion 5 concludes the conclusion.

\section{SYSTEM MODEL}

This portion effectively determines the analytic work in distinction to estimate cognitive radio performance; whereas the conventional throughputs as well as time sensing characteristics were involved.

\subsection{Network model}

Cognitive subscribers are appropriated about the radius $\mathrm{R}$ with respect to band manager.Typically the cognitive secondary user density is $\mathrm{M}$ and the sum of co-operative users $\mathrm{U}$ as determined by the:

$$
\mathrm{U}=\mathrm{M} \cdot \pi \mathrm{R}^{2}
$$




\subsection{Co-operation}

In typical cooperative spotting all the cognitive subscribers exist within the radius $\mathrm{R}$ relaying the regular local-decisions in distinction to the conventional band-manager by means of compelling control channel. Consider the transmission-order as constant be forehand, as well as the cognitive SUs convey their sensing outcomes in sequence order.

Then the terminal results are distinguished with threshold in band. The presence or absence of cognitive PUs judgment is depends on threshold operations and mechanism. The co-operative sensing has accomplished by judgment result in consideration with band manager for all the users.

\subsection{Spectrum Sensing}

This particular portion represents the analysis on the co-operative sensing regarding spectrum for the CRNs. The effective studies of efficient spectrumsensing models are conferred and also co-operative sensing principle is recommended and adopted. In virtue to prevent the unwanted interference to licensed users, the CR is essential of sensing the availability frequency bands in spectrum. The spectrum sensing results in analyzed and to decide among the subsequent multiple hypotheses:

$\mathrm{Hs}_{0}: \mathrm{s}(\mathrm{n})=\mathrm{c}(\mathrm{n})$

$\mathrm{Hs}_{1}: \mathrm{s}(\mathrm{n})=\mathrm{c}(\mathrm{n})+\mathrm{d}(\mathrm{n})$

$\mathrm{n}=1,2, \ldots \ldots \mathrm{K}$

Whereas $s(n)$ the is signal that received at cognitive unlicensed user (secondary), $\mathrm{d}(\mathrm{n})$ is the primary user transmitted signal with mean $\delta_{\mathrm{s}}=0$ and the variance $\tau_{s}^{2}, c(n)$ is AWGN (Additive white Gaussian noise) by accompanying mean $\delta_{\mathrm{w}}=0$, moreover the variance $\tau_{\mathrm{w}}^{2}$, furthermore the $\mathrm{K}$ stand for sum of samples.

\subsection{Local Sensing Mechanism}

We primarily consider the local sensing at each secondary-users and the test statistics $\left(\mathrm{T}_{\mathrm{st}}\right)$ utilizing the energy spotting is indicated by

$\mathrm{T}_{\mathrm{st}}=\sum_{\mathrm{n}=1}^{\mathrm{K}}|\mathrm{S}(\mathrm{n})|^{2}$

If, sum of the samples $\mathrm{K}$ is considerably enough and we adopt the theorem of central-limit to proximate the test statistics as the Gaussian.

$\mathrm{T}_{\mathrm{st}} \mid \mathrm{Hs}_{0} \sim \mathrm{K}\left(\mathrm{K} \tau_{\mathrm{W}}^{2}, 2 \mathrm{~K} \tau_{\mathrm{W}}^{4}\right)$

$\mathrm{T}_{\mathrm{st}} \mid \mathrm{Hs}_{0} \sim \mathrm{K}\left(\mathrm{K} \tau_{\mathrm{S}}^{2}+\tau_{\mathrm{W}}^{2}\right), 2 \mathrm{~K}\left(\tau_{\mathrm{S}}^{2}+\tau_{\mathrm{W}}^{2}\right)^{2}$

Whereas the $(\delta, \tau)$ stand for the Gaussiandistributionin consideration to mean $\delta$ as-wellasvariancet.There upon the probability of false alarm $\mathrm{P}_{\mathrm{fa}}$ and detection probability $\mathrm{P}_{\mathrm{dt}}$ is estimatedas

$$
\begin{aligned}
& P_{\mathrm{fa}}=B\left(\frac{\beta-\mathrm{K} \tau_{\mathrm{W}}^{2}}{\sqrt{2 \mathrm{~K} 4 \tau_{\mathrm{W}}^{4}}}\right) \\
& \mathrm{P}_{\mathrm{dt}}=\mathrm{B}\left(\frac{\beta-\mathrm{K}\left(\tau_{\mathrm{S}}^{2}+\tau_{\mathrm{W}}^{2}\right)}{\sqrt{2 \mathrm{~K}\left(\tau_{\mathrm{S}}^{2} \tau_{\mathrm{W}}^{2}\right)^{2}}}\right)
\end{aligned}
$$

Here $\beta$ implies the typical threshold. If the $\mathrm{P}_{\mathrm{fa}}$ is determined, $\beta$ is defined from the (7) by

$\beta=\sqrt{2 \mathrm{KV}_{\mathrm{W}}^{4}} \mathrm{~B}^{-1}\left(\mathrm{P}_{\mathrm{fa}}\right)+\mathrm{K} \tau_{\mathrm{W}}^{2}$

For the objective possibilities $\left(\mathrm{P}_{\mathrm{fa}}, \mathrm{P}_{\mathrm{dt}}\right)$; the sum of essential samples to attain these objectives is correlated to the $\mathrm{SNR}=\tau_{\mathrm{S}}^{2} / \tau_{\mathrm{W}}^{2}$ has followingK $=$ $2\left[\frac{\mathrm{B}^{-1}\left(\mathrm{P}_{\mathrm{fa}}\right)-\mathrm{B}^{-1}\left(\mathrm{P}_{\mathrm{dt}}\right) \cdot(\mathrm{SNR}+1)}{\mathrm{SNR}}\right]^{2}$

\subsection{Cooperative Sensing}

In Co-operative conventional Sensing, the effective local-decisions by considering the secondary users is possessed at the band-manager through the dedicated control-channel and also test-statistics in distinction to Co-operative Sensing as represented by the summation in regards with each $\mathrm{T}$ as subsequent:

$\mathrm{T}=\sum_{\mathrm{i}=1 .}^{\mathrm{S}} \cdot \sum_{\mathrm{n}=1}^{\mathrm{K}}|\mathrm{S}(\mathrm{n})|^{2}$

In this specific paper, we consider the SNR values of the cognitive SUs are identical, particularly $\tau_{\mathrm{s}, \mathrm{i}}^{2} \tau_{\mathrm{s}}^{2} \forall_{\mathrm{i}}, \tau_{\mathrm{w}, \mathrm{i}}^{2} \tau_{\mathrm{w}}^{2} \forall_{\mathrm{i}}$. In such scenario; central limit theorem is used for dynamic sensing, we may proximate the typical test statistics as the Gaussian

$$
\begin{aligned}
& \mathrm{T}_{\mathrm{st}} \mid \mathrm{Hs}_{0} \sim \mathrm{K}\left(\mathrm{KV} \tau_{\mathrm{W},}^{2} 2 \mathrm{KN} \tau_{\mathrm{W}}^{4}\right) \\
& \left.\mathrm{T}_{\mathrm{st}} \mathrm{Hs}_{1} \sim \mathrm{K}\left(\mathrm{KV} \tau_{\mathrm{S}}^{2}+\tau_{\mathrm{W}}^{2}\right), 2 \mathrm{KV}\left(\tau_{\mathrm{S}}^{2}+\tau_{\mathrm{W}}^{2}\right)^{2}\right) \\
& \mathrm{P}_{\mathrm{fa}} \text { and } \mathrm{P}_{\mathrm{dt}} \text { is estimated as } \\
& \mathrm{P}_{\mathrm{fa}}=\mathrm{B}\left(\frac{\left.\beta-\mathrm{KV} \tau_{\mathrm{w}}^{2}\right)}{\sqrt{2 \mathrm{KV}} \tau_{\mathrm{w}}^{4}}\right) \\
& \mathrm{P}_{\mathrm{dt}}=\mathrm{B}\left(\frac{\beta-\mathrm{KV}\left(\tau_{\mathrm{S}}^{2}+\tau_{\mathrm{W}}^{2}\right)}{\sqrt{2 \mathrm{KV}\left(\tau_{\mathrm{S}}^{2}+\tau_{\mathrm{W}}^{2}\right)^{2}}}\right)
\end{aligned}
$$

The conventional threshold $\beta$ as characterized by the approximated $\mathrm{P}_{\mathrm{fa}}$ as

$\beta=\sqrt{2 \mathrm{KV}_{\mathrm{W}}^{4}} \mathrm{~B}^{-1}\left(\mathrm{P}_{\mathrm{fa}}\right)+\mathrm{KV} \tau_{\mathrm{W}}^{2}$

If we presume the minimal numbers of the samples for attaining the desired $\mathrm{P}_{\mathrm{fa}}$ and $\mathrm{P}_{\mathrm{dt}}$ is estimated by the

$\mathrm{K}=\frac{2}{\mathrm{~V}}\left[\frac{\mathrm{B}^{-1}\left(\mathrm{P}_{\mathrm{fa}}\right)-\mathrm{B}^{-1}\left(\mathrm{P}_{\mathrm{dt}}\right) \cdot(\mathrm{SNR}+1)}{\mathrm{SNR}}\right]^{2}$

2.6 Co-operation overhead:

A co-operation overhead commonly increases with cooperating users count due to the increasedvolume regarding data which is essential to reported and also be handled by band manager. Accordingly, there exists the trade-off at intervals of localprocessing overhead as well as co-operation overhead has they, adds to total sensing-time $\mathrm{T}$. In this particular paper, the $\mathrm{T}$ as add to the local sensing-time $\mathrm{T}_{\text {st }}$ moreover the specific co-operation overhead $T_{Z}$. Hence the achievable total sensingtime $\mathrm{T}$ is characterized by

$$
\mathrm{T}=\mathrm{T}_{\mathrm{Z}+} \mathrm{T}_{\mathrm{St}}
$$

Local sensing-time $\mathrm{T}_{\mathrm{st}}$ is noticed as:

$\mathrm{T}_{\mathrm{St}}=\mathrm{t}_{\mathrm{si}} \mathrm{K}$

$\mathrm{T}_{\mathrm{Sm}}=\frac{2 \mathrm{t}_{\mathrm{Si}}}{\mathrm{v}} \cdot\left[\frac{\mathrm{B}^{-1}\left(\mathrm{P}_{\mathrm{fa}}\right)-\mathrm{B}^{-1}\left(\mathrm{P}_{\mathrm{dt}}\right) \cdot(\mathrm{SNR}+1)}{\mathrm{SNR}}\right]^{2}$

Here $t_{s i}$ is sampling interval and $T_{s m}$ is minimum 
sensing-time in consideration to attain the possibilities $\mathrm{P}_{\mathrm{fa}}$ and $\mathrm{P}_{\mathrm{dt}}$.

Thence; number of the users $\mathrm{V}$ as represented in $\mathrm{Eq}(1), \mathrm{T}_{\mathrm{s}}$ is characterized as radius function $\mathrm{R}$ as follows:

$\mathrm{T}_{\mathrm{S}}=\frac{2 \mathrm{t}_{\mathrm{Si}}}{\mathrm{M} \pi} \cdot\left[\frac{\mathrm{B}^{-1}\left(\mathrm{P}_{\mathrm{fa}}\right)-\mathrm{B}^{-1}\left(\mathrm{P}_{\mathrm{dt}}\right) \cdot(\mathrm{SNR}+1)}{\mathrm{R} \cdot \mathrm{SNR}}\right]^{2}$

In this research work, we considered only the propagation time that reports the conventional local-decisions to the band manager has cooperation overhead and we-deal with the SUs identically distributed over the band-manager. As the result, the typical average-distance in distinction to band-manager in the direction of secondary-user is been estimated as $/ \sqrt{ } 2$.Formerly the specific cooperation overhead as peruser has represented has:

$\overline{\mathrm{T}}_{\mathrm{Z}}=\frac{\mathrm{R}}{\sqrt{2} \mathrm{Z}}$

Here, the ' $Z$ 'as considered to be speed of the light. Accordingly,the co-operation overhead regarding the secondary-network is characterized as

$$
\begin{aligned}
\mathrm{T}_{\mathrm{Z}} & =\mathrm{V} \overline{\mathrm{T}}_{\mathrm{Z}} \\
\mathrm{T}_{\mathrm{Z}} & =\mathrm{V} \cdot \frac{\mathrm{R}}{\sqrt{2} \mathrm{Z}} \\
\mathrm{T}_{\mathrm{Z}}=\frac{\mathrm{M} \pi \mathrm{R}^{3}}{\sqrt{2} \mathrm{Z}} &
\end{aligned}
$$

Categorically; number of the users ' $\mathrm{V}$ ' is assessed as the (1), and if $T_{z}$ declines the users count ' $V$ ' increases, also when the $\mathrm{T}_{\mathrm{z}}$ increases the ' $\mathrm{V}$ ' also increases.

By considering (19), conventionally the total sensing-time ' $\mathrm{T}$ ' be written as

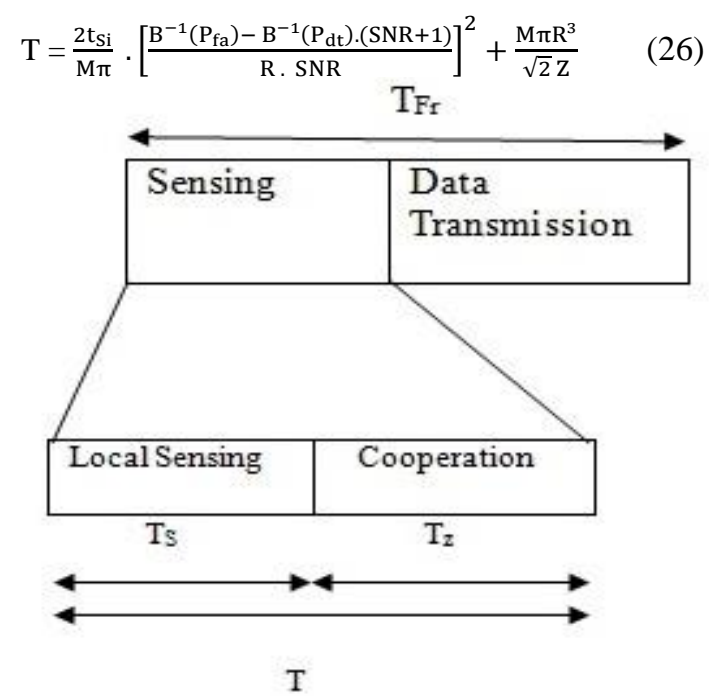

Fig 1. Transmission and reception frame in distinction to secondary networks

\section{SECONDARY NETWORKS THROUGHPUT}

In this portion, we interpret the total sensing-time effect on throughput in consideration to secondary wireless-system. Fig.1 interprets the transmission and reception mechanism of specific secondary system considered in this particular research work.

In each duration frame $\mathrm{T}_{\mathrm{Fr}}$, the unlicensed users senses the channels for duration of the $T_{z}$ noticed in by (22). After all, the SUs transmit their particular local-decisions to the band manager considering the duration of the $T_{z}$. If no PUs is spotted in channel, the SUs can utilize rest of the frame $T_{F r}-\left(T_{Z}+T_{z}\right)$ in consideration to transmission of data. If the active PU is spotted, the cognitive SUs will not transfer in frame as well as waits until the subsequent frame when the channel sensing is repeated. we assume communication frame length of the primary-system has $\mathrm{T}_{\mathrm{PRF}}$, we assume the secondary network throughput to data which is able to disseminated when the primary-network as no transmission and reception mechanism during the $\mathrm{T}_{\mathrm{PRFr}}+\mathrm{T}_{\mathrm{Fr}}$.

Let determine $\mathrm{P}_{\mathrm{z}}$ has the possibility which PU doesn't start the transmission during the $\mathrm{T}_{\mathrm{PRFr}}+\mathrm{T}_{\mathrm{Fr}}$ and normalized through-put of the secondary network is characterized as

$\mathrm{Th}=\frac{\mathrm{T}_{\mathrm{Fr}}-\mathrm{T}}{\mathrm{T}_{\mathrm{Fr}}}\left(1-\mathrm{P}_{\mathrm{fa}}\right) \mathrm{P}_{\mathrm{z}}$

In case the cognitive PUs has an conventional exponential on-off typical traffic model, moreover the mean-durationregarding periods defined by the $\gamma$, and $\mathrm{P}_{\mathrm{Z}}$ represented by the:

$\mathrm{P}_{\mathrm{z}}=\exp \left(-\frac{\mathrm{T}_{\mathrm{PRFr}}+\mathrm{T}_{\mathrm{Fr}}}{\gamma}\right)$

By considering the duration of frame $\mathrm{T}_{\mathrm{PRFr}}, \mathrm{T}_{\mathrm{Fr}}$ moreover the possibility of false alarm $\mathbf{P}_{f a}$ has fixed, and secondary networks normalized throughput of is determined by:

$\mathrm{Th}=\frac{\mathrm{T}_{\mathrm{Fr}}-\mathrm{T}}{\mathrm{T}_{\mathrm{Fr}}}\left(1-\mathrm{P}_{\mathrm{fa}}\right) \exp \left(-\frac{\mathrm{T}_{\mathrm{PRFr}+\mathrm{T}_{\mathrm{F}} \mathrm{r}}}{\gamma}\right)$

\section{SIMULATION ANALYSIS}

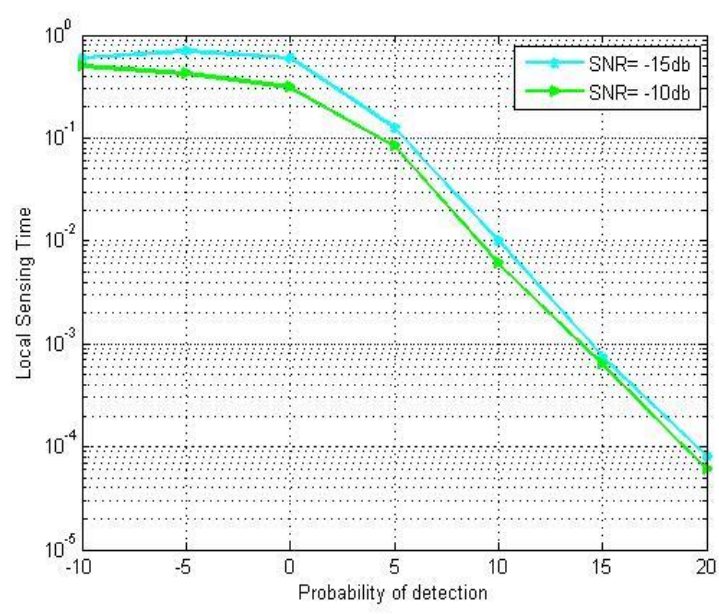

Fig 2. Local Sensing Time versus Detection probability

The MATLAB is adopted simulation results; the above figure characterizes the relation at intervals of local sensing time and possibility of detection at SNR $=[-15 \mathrm{db}, 10 \mathrm{db}]$, need the less sensing time in case of detection mechanism to enhance the spectrum usage and also the throughput in network improves as detection probability increases, for average intervals of time among the secondary networks sensing time, 
moreover the local sensing time is compared with detection process at different SNR.

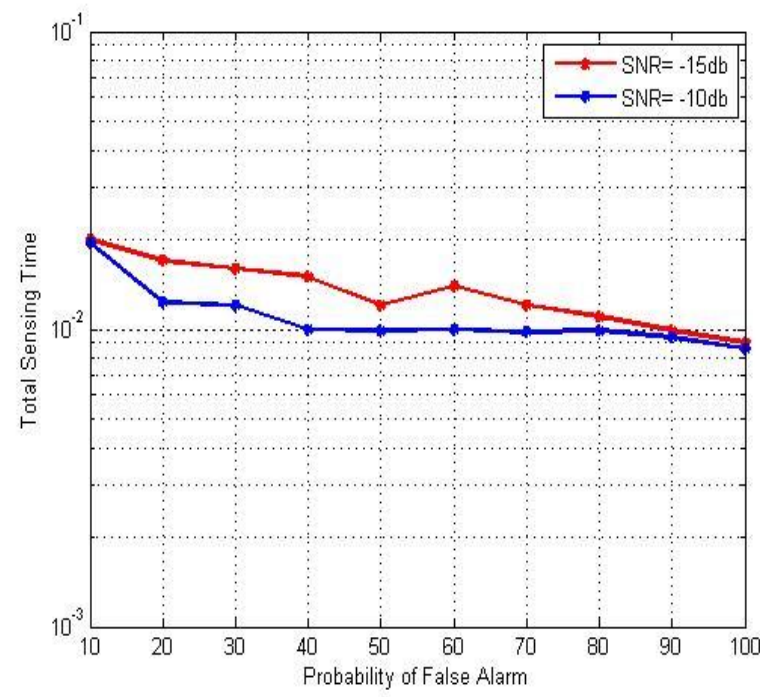

Fig 3. Total Sensing Time versus False Alarm probability

Fig 3 illustrates the throughput in network with gradual decrease as false alarm possibility increases, the outcome means the secondary networks throughput declines because of the co-operation overhead effect and total sensing time is correlated with false alarm at $\mathrm{SNR}=-15 \mathrm{db}$ and $\mathrm{SNR}=-10 \mathrm{db}$, this research work characterizes and gives a effective analysis throughput of secondary network. This ultimately improves the sensing mechanism and takes low time to sense the gives either the cognitive users are absence or presence by measuring the received signal power.

\section{CONCLUSION}

In this investigative work, the co-operation overhead effect on secondary network throughput has observed and analyzed. By considering delay of propagation we have determined the secondary network performance. The total sensing-time as essential for particular detection conditions (target false alarm and detection probability) as well as the typical secondary-networks throughput has derived. Practical results has interpreted that sensing ability decreases because of increase in co-operation overhead has sum of the cooperation users gradually increases. Furthermore, the decrease in throughput gradually decrease the sensing ability as in such case when increase in cooperation overhead. In CRNs, the SUs as authorization to use the bands in case the PUs were not using it. For supporting of functionality of spectrum reuse, the cognitive SUs need to sense the conditions of radio frequency, and if the primary users is came active ,the SU need to vacate the frequency band in specific amount of the time. Thence, spectrum sensing mechanism is a crucial in CRNs and detection and false alarm probabilities are two important criteria's in spectrum sensing process. The primary users were protected in case were higher the detection possibility, from the perspective of secondary users, false alarm probability, the most chances the band is reutilized when in available condition, thus the higher attainable through-put for secondary network.

\section{REFERENCES}

[1]. Binshen, Longyang Huang and Chengshi Zhao 2008, Energy Detection Based Spectrum sensing for Cognitive Radios in Noise of uncertainty, IEEE International Conference,pp.12-18.

[2]. Y.-J Choi, W.Pak, Y.Zin and S.Rangarajan; "Throughput analysis of cooperative spectrum sensing in Rayleigh-faded cognitive radio systems". IET Communications, Volume: 6, Issue: 9 , June 14 2012,pp. $1104-1110$.

[3]. B. Wang and K. Liu, "Advances in Cognitive radio networks: A survey," Selected Topics in Signal Processing, IEEE Journal, 5(1)(2011) 5-23.

[4]. B. Wang Yun Han Bae, Jung Woo Baek; "Achievable Throughput Analysis of Opportunistic Spectrum Access in Cognitive Radio Networks With Energy Harvesting”. IEEE Transactions on Communications, Volume: 64, Issue: 4 , April 2016, pp. $1399-1410$.

[5]. Hyun-Ho Choi et all; "Throughput analysis and optimization of distributed collision detection protocols in dense wireless local area network", Journal of Communications and Networks Volume: 18 , Issue: 3 , June 2016,pp. $502-512$.

[6]. S. Haykin, D. Thomson, and J. Reed, "Spectrum sensing for cognitive radio, "Proceedings of the IEEE, Invited Paper, 97(5) (2009) 849-877.

[7]. Binshen, Longyang Huang and Chengshi Zhao 2008, Energy Detection Based Spectrum sensing for Cognitive Radios in Noise of uncertainty, IEEE International Conference,pp.12-18.

[8]. Weiwei Cui ; Haifeng Zhao; Xiaomin Mu; "Throughput Analysis in Cooperative Sensing Networks over Imperfect Reporting Channel", 2011 Third International Conference on Communications and Mobile Computing, 18-20 April 2011.

[9].Kwang-Cheng Chen, Irving T. Ho, "Cognitive Radio Networks", CTiF Workshop 2007.

[10]. S. S. Alam, L. Marcenaro and C. Regazzoni "Opportunistic Spectrum Sensing and Transmissions" Cognitive Radio and Management: Technonlogy and Strategy. IGI Global,(2013) 1-28.

[11]. Akyildiz, F.Lee, Vuran and Mohanty(2006), "Next Generation/dynamic spectrum access cognitive radio wireless networks:vol.50,IEEE 2017-2159. Thesis:

[12]. Mitola.J (2000). Cognitive radio-an integrated agent architecture for software defined radio, Ph.D. thesis, Royal institute of Technology(KTH) 